\title{
Respuesta agronómica de mucilago de cacao (Theobroma cacao L.) en cultivo de maíz (Zea mays L.)
}

\author{
Agronomic response of cocoa mucilago \\ (Theobroma cacao L.) in maize culture (Zea mays L.)
}

\author{
Diana María Sánchez Olaya ${ }^{a^{*}}$ \\ Wilson Rodríguez Pérez \\ Diego Francisco Castro Rojas ${ }^{\mathrm{c}}$ \\ Edwin Trujillo Trujillo ${ }^{d}$
}

Fecha de Recepción: 25.04.18

Fecha de aceptación: 10.06.19

DOI: https://doi.org/10.19053/01217488.v10.n2.2019.7958

\begin{abstract}
Resumen
Se comparó la producción de mucilago drenado de granos de Theobroma cacao, $T$. grandiflorum y $T$. bicolor en caja de madera y luego se evaluó su posible uso como biofertilizante. T. cacao presentó la mayor producción de mucilago drenado ( $4.5 \mathrm{ml}$ mucilago $\mathrm{kg}^{-1}$ grano h$^{-1}$ ) con contenidos de $11.18 \mathrm{mg} \mathrm{P} \mathrm{L.}^{-1}, 0.2 \%$ $\mathrm{K}$ y $0.08 \% \mathrm{~N}$. Posteriormente se determinó el crecimiento de dos variedades de Zea mays (ICA V-305 y Calilla) usando mucilago drenado de T. cacao. El experimento en campo tuvo cinco tratamientos (fertisun, control (sin abonado), $5 \%$ mucilago, $10 \%$ mucilago y $15 \%$ mucilago) bajo un diseño completamente al azar en arreglo de parcelas divididas. Las variables de respuesta agronómica evaluadas fueron: TAC y TRC. En la evaluación de TAC en las dos variedades de Zea mays estudiadas se observó que la variedad calilla presentó mayor crecimiento en menor tiempo cuando se aplicó mucilago drenado al $5 \%$ respecto de la variedad ICA V-305, por lo cual se consideró el mejor tratamiento para usar como abono líquido. Considerando la existencia de malezas en el sitio de estudio, las cuales fueron retiradas solo en los dos primeros muestreos (13 y 21 dds) no se observó una tendencia clara en los valores TRC para las dos variedades de Z. mays evaluadas.
\end{abstract}

Palabras clave: mucilago drenado, análisis de crecimiento, granos de cacao, abono.

a "Magister en Agroforesteria, Programa de Ingeniería Agroecológica, Facultad de Ingenierías, Universidad de la Amazonia. Autor de correspondencia: dia.sanchez26@gmail.com

b Magister en Ciencias-Química, Programa de Química, Facultad de Ciencias Básicas, Universidad de la Amazonia

c Ingeniero Agroecólogo, Programa de Ingeniería Agroecológica, Facultad de Ingenierías, Universidad de la Amazonia

d Ingeniero Agroecólogo, Programa de Ingeniería Agroecológica, Facultad de Ingenierías, Universidad de la Amazonia 


\begin{abstract}
The production of drained mucilage of beans of Theobroma cacao, T. grandiflorum and T. bicolor in wooden box was compared and then its possible use as biofertilizer was evaluated. T. cacao showed the highest production of drained mucilage $\left(4.5 \mathrm{ml}\right.$ mucilage $\mathrm{kg}^{-1}$ grain $\mathrm{h}^{-1}$ ) with contents of $11.18 \mathrm{mg} \mathrm{P} \mathrm{L}$. 1, $0.2 \% \mathrm{~K}$ and $0.08 \% \mathrm{~N}$. Subsequently the growth of two varieties of Zea mays (ICA V-305 and Calilla) using drained mucilage of T. cacao was characterized. The field experiment had five treatments (fertisun, control (without fertilizer), 5\% mucilage, 10\% mucilage and 15\% mucilage) under a completely random design in divided plots. The agronomic response variables evaluated were: Absolute Growth Rate (AGR) and Relative Growth Rate (RGR). In the evaluation of AGR in the two varieties of Z. mays studied, it was observed that the Calilla variety showed greater growth in less time when applied 5\% mucilage with respect to the variety ICA V-305, for which it was considered the best treatment to use as biofertilizer. Considering the existence of weeds in the study site, which were only removed in the samplings at 13 and 21 days after seedling not clear tendency was observed in the RGR values for the two varieties of $Z$. mays evaluated.
\end{abstract}

Key words: drained mucilage, growth analysis, cocoa beans, fertilizer.

\title{
1. INTRODUCCIÓN
}

El cacao es usado principalmente en la elaboración de chocolates [1], siendo a nivel mundial, Costa de Marfil el mayor productor de grano de cacao. A nivel latinoamericano, Colombia ocupa el cuarto lugar entre los países productores y a nivel nacional, el departamento de Santander concentra la mayor producción de grano de cacao [2]. De otro lado, la explotación comercial de cacao genera grandes cantidades de grano, pulpa y cáscara, sin embargo una vez obtenido el grano seco de cacao, los coproductos que permanecen son conformados por principalmente por tres fracciones: mazorca del cacao, cáscara del grano de cacao y mucilago o pulpa de cacao; en muchos casos, estos coproductos son sub-explotados y considerados desechos no deseables de la industria del cacao/ chocolate [3]. La cáscara de cacao presenta un uso potencial en la producción de pectinas $[4,5]$, alimentación animal [6]. La pulpa o mucílago se usa en la producción de bebidas alcohólicas [7] y jalea [8]. Un estudio en Ecuador evidenció que el $81 \%$ de los agricultores del sector cacaotero no potencializan el desarrollo técnico en la cadena de valor de este producto, presentándose un desperdicio del mucílago de cacao del orden del $72 \%$ por desinterés en la innovación agrícola [9]. Es de notar que en Colombia, se regula la producción de fertilizantes $\mathrm{y}$ acondicionadores de suelos mediante resolución 00150 de 2003 del Instituto Colombiano Agropecuario (ICA) [10] y la Norma Técnica Colombiana 5167 [11], además se encuentra la resolución 000187 de 2006 del
Ministerio de Agricultura y Desarrollo Rural [12] que reglamenta el uso de los productos agropecuarios ecológicos y la resolución 000698 de 2011 del ICA [13] que establece los requisitos de ensayos de eficacia de insumos de uso agrícola. Se han registrado estudios relacionados con aplicaciones agronómicas de los residuos de cacao. Al evaluar el efecto del vertimiento de subproductos del beneficio del cacao en el suelo no se encontró una relación directa respecto de si eran las cáscaras o el exudado, el responsable de las variaciones en los parámetros evaluados [14]. Al evaluar la fitotoxicidad de mucílago de cacao más abono líquido de hojas sobre diferentes especies en post emergencia en malezas de 30 días, se observó que la mortalidad de malezas fue mayor en el tratamiento de mucílago de cacao al $100 \%$ [15]. El abono líquido como biofertilizante y bioestimulante foliar de origen orgánico y de producción casera, se constituye en una alternativa al alcance de los productores y es importante en la producción con orientación ecológica de cultivos [16]. Los fertilizantes de origen orgánico contienen macronutrientes y micronutrientes en una relación más estable que permiten desarrollar de forma más rápida y completa las plantas, en comparación con la fertilización química cuya composición está basada principalmente de elementos mayores (NPK) de alta solubilidad [17]. No obstante, el uso desbalanceado y frecuente de estos agroquímicos han contaminado en gran extensión el medio ambiente [18]. Las semillas de cacao están rodeadas de un mucílago que 
contiene de 10 a $15 \%$ de azúcar, $1 \%$ de pectina y $1.5 \%$ de ácido cítrico. Parte de este mucílago o pulpa es necesaria para la producción de alcohol y ácido acético en la fermentación de las almendras, pero, entre el 5 a $7 \%$ drena como exudado [19]. Normalmente se desperdician más de 70 litros por tonelada de este material mucilaginoso. Este exceso de pulpa ha sido utilizado para fabricar productos alimenticios [20]. Lo anterior evidencia la necesidad de seguir evaluando el potencial uso de los residuos del cacao dentro del contexto de la innovación agrícola para mejorar los ingresos de los agricultores. De otro lado, el maíz es uno de los cereales más importantes del mundo después del trigo y arroz en términos de área cultivada y producción total, siendo muy usado en alimentación humana y animal [21]. Así, el propósito del presente trabajo fue evaluar en campo la respuesta agronómica de maíz (Zea mays L.) usando como biofertilizante el mucilago de cacao.

\section{MATERIALES Y MÉTODOS}

\section{1 Área de estudio}

El estudio se realizó en dos sitios: la Granja Experimental Agroecológica Balcanes -(GEAB) y El Centro de Investigaciones AmazónicasMacagual "Cesar Augusto Estrada Gonzales"(C.I. Macagual) de la Universidad de la Amazonia (Florencia, Caquetá). La GEAB presenta coordenadas de $1^{\circ} 37^{\prime} \mathrm{N}$ y $75^{\circ} 36^{\prime} \mathrm{W}$, con una elevación de $250 \mathrm{~m}$, en zona de vida bosque húmedo tropical, con precipitación media anual de $3793 \mathrm{~mm}$, temperatura media anual de $25{ }^{\circ} \mathrm{C}$ y humedad relativa media anual de $84 \%$ [22]. El C.I. Macagual presenta coordenadas $1^{\circ} 37 \mathrm{~N}$ y $75^{\circ} 36 \mathrm{~W}$ con una altura de $300 \mathrm{~m}$ de elevación, con zona de vida bosque tropical húmedo, temperatura media anual de $25.5{ }^{\circ} \mathrm{C}$, humedad relativa media anual de $76.3 \%$ y precipitación media anual de $3793 \mathrm{~mm}$ [23].

\subsection{Recolección de frutos}

En la GEAB, se cosecharon frutos de cacao ( $T$. cacao L.) y maraco (T. bicolor Bonpl.) en el mes de octubre de 2014 en un sistema agroforestal, conformado por caucho (Hevea brasiliensis (Willd. ex A. Juss.) Müll. Arg.), cacao (T. cacao L.), maraco (T. bicolor Bonpl.), copoazú ( $T$. grandiflorum (Willd. ex Spreng.) K. Schum.), borojo (Borojoa patinoi Cuatrec.) y arazá (Eugenia stipitata $\mathrm{McVaugh}$ ). En el C.I. Macagual se cosechó frutos de copoazú (T. grandiflorum) de un sistema de monocultivo en el mes de abril de 2015. Se realizó la cosecha de mazorcas sanas y maduras en forma aleatoria mediante recorrido en $z i g-z a g$ en los sitios de muestreo.

\subsection{Estado de madurez del fruto}

Las mazorcas cosechadas se pesaron por separado y se despulparon, luego se pesaron los granos obtenidos y se determinó sobre el mucílago los siguientes parámetros: Color (organoléptico), $\mathrm{pH}$ (potenciometría), ${ }^{\circ}$ Brix a 20 ${ }^{\circ} \mathrm{C}$ (refractometría), acidez titulable (titulación acido base) e índice de madurez (Brix/acidez titulable) [24].

\subsection{Rendimiento de mucilago}

Los granos de cacao se llevaron a cajas de madera de dimensiones $0.5 \mathrm{~m}$. x $0.5 \mathrm{~m}$. x $1 \mathrm{~m}$. donde la base de la caja de madera, se encontraba con una inclinación de $15^{\circ}$ para facilitar el escurrimiento del mucilago a través de un orificio [25]. Se registró cada hora, el volumen de mucilago obtenido en una probeta para determinar la velocidad de producción de mucilago y se relacionó a la masa de grano usado $\left(\mathrm{L} \mathrm{kg}^{-1} \mathrm{~h}^{-1}\right)$. El mucilago obtenido se conservó en refrigeración a $4{ }^{\circ} \mathrm{C}$ hasta el momento de su uso.

\subsection{Análisis del mucilago}

Sobre el mucílago obtenido se determinó: $\mathrm{pH}$ (potenciometría), ${ }^{\circ}$ Brix (refractometría), acidez titulable (valoración acido-base) [24], conductividad eléctrica (conductivímetro) [26], cenizas (calcinación $550{ }^{\circ} \mathrm{C}$ por $4 \mathrm{~h}$ ), nitrógeno total (Kjeldahl), humedad (horno de secado), materia seca (estimación), fósforo total (molibdovanadato), potasio (digestión ácida, absorción atómica) [27], peso específico (Picnómetro) [28], carbono (Walkley-Black) [29].

\subsection{Análisis de suelos}

Se obtuvo muestra compuesta de suelo y se determinó densidad aparente (cilindro), textura 
(Bouyoucos), color (tabla munsell), resistencia (penetrometro de impacto), $\mathrm{pH}$ en agua (1:1) (potenciometria), capacidad de intercambio catiónico (acetato de amonio 1M y pH neutro) y carbono orgánico (Walkley-Black) [30].

\subsection{Respuesta agronómica}

Se siguió la metodología modificada de Di Rienzo, et al. [31], donde se utilizó como material vegetal las semillas de maíz (Zea mays L.), de las variedades ICA V-305 y Calilla, las cuales fueron evaluadas bajo un diseño completamente al azar con cinco repeticiones, en arreglo de parcelas divididas, donde la parcela principal correspondió a las variedades de maíz $\left(100 \mathrm{~m}^{2}\right)$ y las subparcelas a los abonos foliares evaluados $\left(20 \mathrm{~m}^{2}\right)$. Los abonos foliares (tratamientos) evaluados fueron: dilución acuosa de fertisun ${ }^{\circledR}$ $1 \%(\mathrm{v} / \mathrm{v})$, diluciones acuosas de mucilago de cacao al $5 \%(\mathrm{v} / \mathrm{v}), 10 \%(\mathrm{v} / \mathrm{v})$ y $15 \%(\mathrm{v} / \mathrm{v})$. El control correspondió a las dos variedades de maíz evaluadas sin abonado (Figura 1).

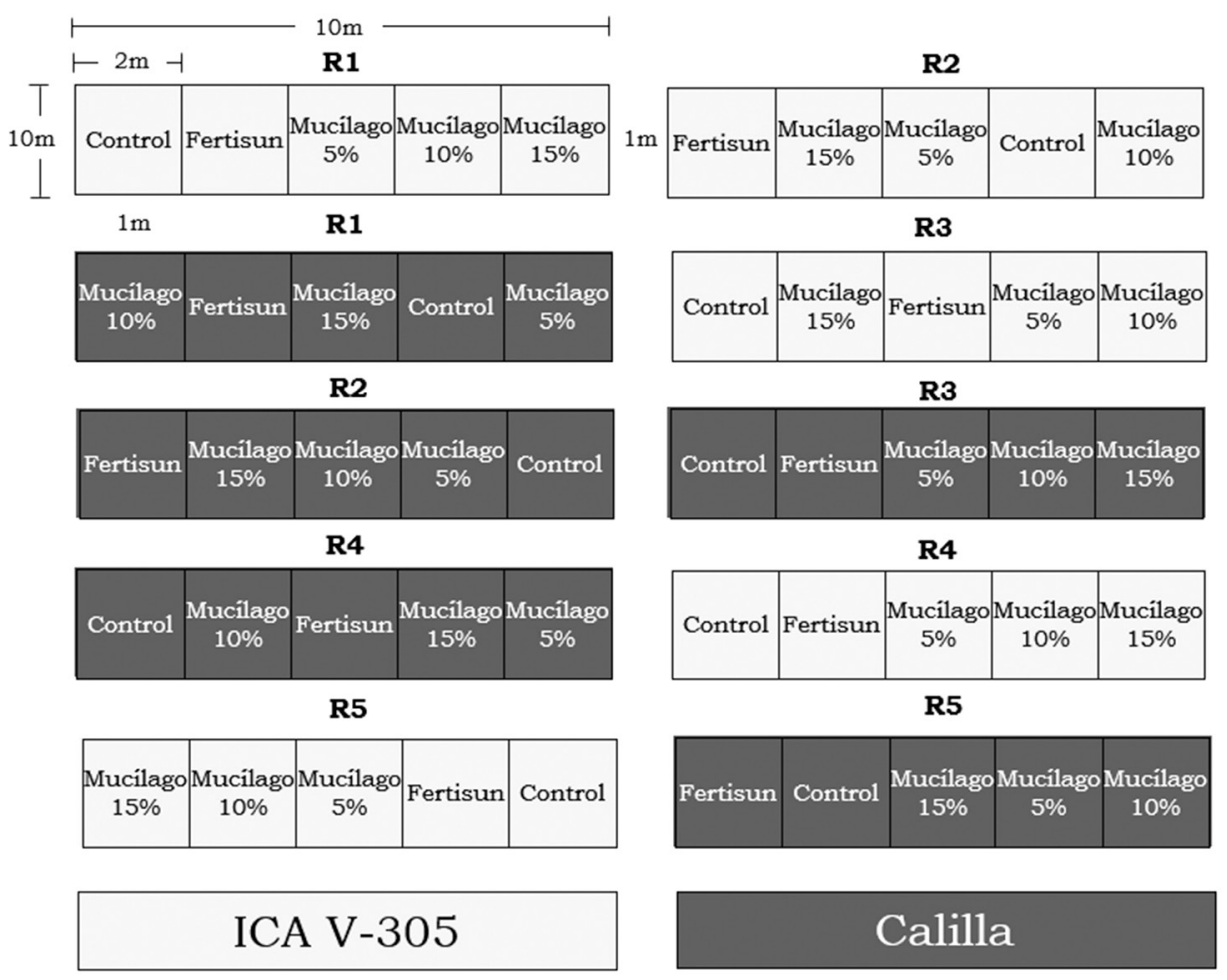

Figura 1. Distribución de los abonos foliares y variedades de maíz, evaluados en un diseño completamente al azar en arreglo de parcelas divididas.

Para los tratamientos con mucilago de cacao fue utilizada una dosis de $50 \mathrm{ml}, 100 \mathrm{ml}$ y $150 \mathrm{ml}$ por cada $1000 \mathrm{ml}$ de agua, respectivamente, estás dosis fueron aplicadas en horas de la mañana (antes de las 9:00 am) durante tres períodos de abonado, con una frecuencia de 15 días. La densidad de siembra utilizada fue de 3 plantas $\mathrm{m}^{-2}$.
Los muestreos destructivos se realizaron en tres plantas por repetición y por tratamiento, cada 8 días a partir de los 13 días después de siembra (dds), con el fin de determinar medidas directas de peso fresco $(\mathrm{g})$ y peso seco $(\mathrm{g})$, para posteriormente realizar el cálculo de los índices de crecimiento propuestos por Almanza, et al. [32]: Tasa Absoluta de Crecimiento (TAC) y Tasa Relativa de Crecimiento (TRC) (Tabla 1). 
Diana María Sánchez Olaya et. al.

Tabla 1. Descripción de los índices de crecimiento evaluados [33]

\begin{tabular}{cccc}
\hline Índice & Descripción & Fórmula & Unidades \\
\hline \multirow{2}{*}{ TAC } & Tasa Absoluta de Crecimiento & $\frac{W_{2}-W_{1}}{T_{2}-T_{1}}$ & $\mathrm{~g} \mathrm{día}^{-1}$ \\
\hline TRC & Tasa Relativa de Crecimiento & $\frac{\operatorname{Ln} W_{2}-L n W_{1}}{T_{2}-T_{1}}$ & $\mathrm{~g} \mathrm{día}^{-1}$ \\
\hline
\end{tabular}

$\mathrm{W}_{2}$ : Peso seco final, $\mathrm{W}_{1}$ : Peso seco inicial, $\mathrm{T}_{2}$ : Tiempo final, $\mathrm{T}_{1}$ : Tiempo inicial, Ln: Logaritmo natural

\subsection{Análisis estadístico}

Para los parámetros de estado de madurez del fruto y análisis de mucilago se determinó media y error estándar, luego se sometieron a análisis de varianza y prueba de comparación de medias de LSD Fisher con nivel de confianza $\alpha=0.05$ para determinar diferencias significativas, en el software InfoStat versión 2017. El comportamiento de los índices TAC y TRC se evaluó mediante la técnica de ajuste de curvas en el programa Microsoft Excel 2013 [34].

\section{RESULTADOS Y DISCUSIÓN}

\subsection{Condiciones agroclimáticas del sitio de estudio}

El suelo del sitio de estudio (GEAB) presentó las siguientes características: densidad aparente $1.3 \mathrm{~g} \mathrm{~cm}^{-3}$, textura arcillosa, color amarillo $10 \mathrm{YR}$ 7/6, resistencia $1.4 \mathrm{MPa}, \mathrm{pH} 5.47$, Capacidad Intercambio Catiónico $19.46 \mathrm{meq} / 100 \mathrm{~g}$ y Carbono orgánico 1.3\%. El presente estudio comprendió el período del 18 de agosto al 16 de septiembre de 2015, donde la variación diaria del brillo solar estuvo entre $0-9.9$ horas, evaporación entre 0.7 - $4.3 \mathrm{~mm}$., humedad relativa entre $73-91 \%$, precipitación entre $0-27.5 \mathrm{~mm}$. y temperatura media entre $21.9-27.8^{\circ} \mathrm{C}[35]$.

\subsection{Estado de madurez del fruto}

Al comparar los parámetros de madurez de las pulpas de los frutos evaluados, se estableció diferencia significativa en $\mathrm{pH}$, acidez titulable y ${ }^{\circ}$ Brix. El maraco presentó el mayor valor de índice de madurez (Tabla 2). Los valores de $\mathrm{pH}$ de las pulpas de los frutos evaluados son mayores comparados con Camú Camú (pH 2.56) [36]. El estudio de especies del género Theobroma de la Amazonia colombiana de Melgarejo, et al. [37] registraron valores de $\mathrm{pH}$ de pulpa de copoazú entre 3.30-3.67 y de pulpa de maraco entre 6.0-6.5; valores de acidez titulable de pulpa de maraco entre $0.12-0.14 \%$, de pulpa de copoazú entre 1.36-2.17\%, ${ }^{\circ}$ Brix de pulpa de maraco entre 4.4-11 y de pulpa de copoazú entre 6-15 y finalmente valores de índice de madurez de pulpa de maraco entre 38-110 y de pulpa de copoazú de 410. Estos valores son similares a los registrados en el presente estudio (Tabla 2). Los valores de $\mathrm{pH}$ de la pulpa de cultivares de cacao de Venezuela (3.02-4.2) [38] también son valores similares a los registrados en el presente estudio. La pulpa de cacao de tipo nacional y CCN-51 del Ecuador mostraron valores similares de $\mathrm{pH}$ (3.7$3.87)$, valores ligeramente menores de acidez (0.71-0.91) y valores similares de ${ }^{\circ}$ Brix (15-16) [8] comparado con los valores de los mismos parámetros registrados en el presente estudio.

Tabla 2. Datos de estado de madurez del fruto

\begin{tabular}{lcccc}
\hline \multirow{2}{*}{ Parámetros } & \multicolumn{2}{c}{ Cacao } & \multirow{2}{*}{ Maraco } & \multirow{2}{*}{ Copoazú } \\
\cline { 2 - 3 } & \multicolumn{1}{c}{ Rojo } & Amarillo & & \\
\hline $\mathrm{pH}$ & $4.27 \pm 0.28 \mathrm{a}$ & $3.90 \pm 0.24 \mathrm{a}$ & $6.31 \pm 0.06 \mathrm{c}$ & $4.95 \pm 0.15 \mathrm{~b}$ \\
Acidez titulable (ácido cítrico \%) & $1.20 \pm 0.28 \mathrm{bc}$ & $0.95 \pm 0.29 \mathrm{ab}$ & $0.21 \pm 0.03 \mathrm{a}$ & $1.86 \pm 0.24 \mathrm{c}$ \\
${ }^{\circ}$ Brix $\left(20{ }^{\circ} \mathrm{C}\right)$ & $14.44 \pm 2.57 \mathrm{ab}$ & $18.71 \pm 0.88 \mathrm{~b}$ & $12.0 \pm 0.32 \mathrm{a}$ & $12.77 \pm 1.16 \mathrm{a}$ \\
Índice de madurez & $12.44 \pm 0.98 \mathrm{a}$ & $26.13 \pm 10.88 \mathrm{a}$ & $60.52 \pm 9.29 \mathrm{~b}$ & $8.60 \pm 0.4 \mathrm{a}$ \\
\hline
\end{tabular}

$\mathrm{n}=3$. Letra minúscula diferente en la misma fila indica diferencia significativa. Nivel de significancia 0.05 . 


\subsection{Rendimiento de mucilago}

La cinética de producción de mucílago por los granos de cacao y maraco presento un comportamiento gráfico en forma de hipérbole; en menos de $5 \mathrm{~h}$ se produjo más del $80 \%$ del mucilago. En el caso de copoazú, el mucilago presentó un comportamiento no lineal durante su producción, en las primeras $5 \mathrm{~h}$, sin embargo, solo se produce alrededor del $35 \%$ de mucilago durante ese tiempo (Figura 1). Es de notar, que el copoazú presentó el índice de madurez más bajo asociado a un mayor valor de acidez titulable (Tabla 2).
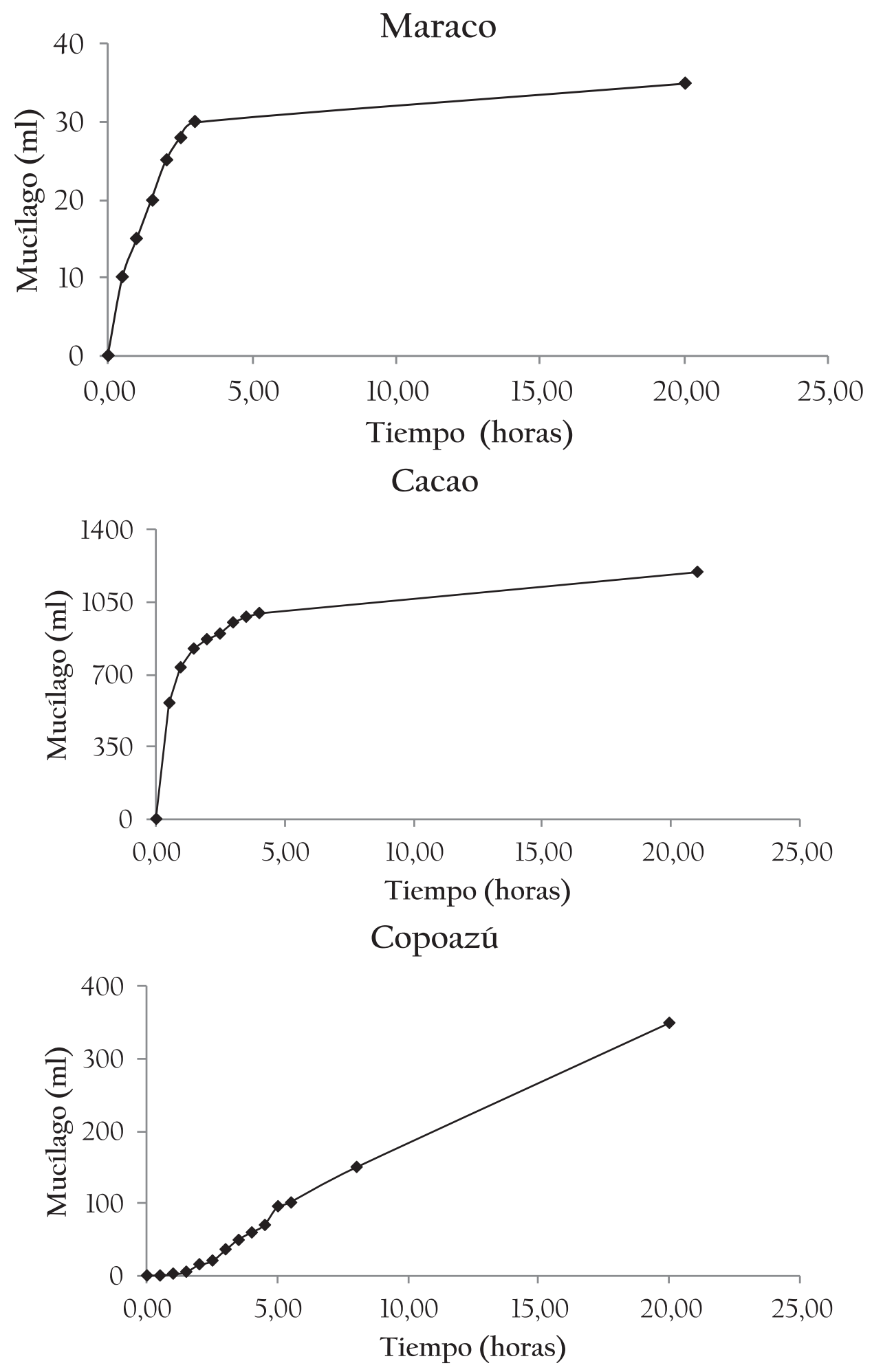

Figura 2. Cinética de la producción de mucilago de grano de cacao, copoazú y maraco. 
El fruto con mayor potencial comercial de producción de mucilago fue el cacao (mezcla de variedades rojo y amarillo) ya que presentó el mayor rendimiento correspondiente a $4.5 \mathrm{ml}$ mucilago $\mathrm{kg}^{-1}$ grano fresco hora-1 , cuyo valor es el doble del mucilago producido por el copoazú y 22.5 veces más del mucilago que produjo el maraco (Tabla 3 ).

Tabla 3. Rendimiento de mucilago ( $\mathrm{ml}$ mucilago $\mathrm{kg}^{-1}$ grano $\mathrm{h}^{-1}$ ) obtenido a partir de cacao, copoazú y maraco.

\begin{tabular}{|c|c|c|c|}
\hline Parámetro & Cacao & Maraco & Copoazú \\
\hline Mazorca $(\mathrm{kg})$ & 61.3 & 25 & 13.8 \\
\hline Grano fresco $(\mathrm{kg})$ & 12.8 & 9.75 & 7.6 \\
\hline Mucilago (ml) & 1.200 & 30 & 350 \\
\hline Tiempo (h) & 21 & 20 & 20 \\
\hline $\mathrm{ml}$ exudado $\mathrm{kg}^{-1}$ grano fresco hora ${ }^{-1}$ & 4.5 & 0.2 & 2.3 \\
\hline
\end{tabular}

Debido al bajo rendimiento de mucilago presentado por el maraco (Tabla 3), este no se consideró en los análisis de parámetros físicos y químicos de mucilago obtenido por escurrimiento de semillas (Tabla 4). En general, se observó diferencia significativa entre los valores de los parámetros evaluados en mucilago de cacao y copoazú. Aunque el mucilago de copoazú tuvo menor rendimiento (Tabla 3) proporciona más nitrógeno y potasio que el exudado de cacao, el cual sobresale por un mayor contenido de fósforo. De otro lado, se esperaría un mayor aporte de minerales del mucilago de cacao considerando su alto contenido de cenizas (Tabla 4). La relación carbono/nitrógeno del mucilago de cacao (24.8) es mayor que el valor dado para la relación de $\mathrm{C} / \mathrm{N}$ de copoazú (12.6), lo cual indica que el mucilago de copoazú es más fácilmente mineralizable [39]. Al comparar los contenidos de N, P y K del mucilago obtenido después de escurrimiento de grano de cacao con abono líquido, se observó que el mucilago de cacao presento un contenido mayor de nitrógeno $(0.08 \%)$ respecto del mismo nutriente en abono líquido fresco (0.06 $\%$ [ [40], menor contenido de nitrógeno respecto del abono líquido supermagro $(0.12 \% \mathrm{~N})$ [41], menor contenido de nitrógeno respecto del abono líquido de bovino $(0.25 \%)$ y de abono líquido de cerdo $(0.41 \%)$ [42]. Con respecto al contenido de fósforo, el mucilago de cacao tiene valores bajos (11.18 $\left.\mathrm{mg} \mathrm{L}^{-1}\right)$ respecto del abono líquido fresco $(0.04 \%)$ [40], valores altos comparado con el abono líquido supermagro (8.6 ppm) [41] y valores bajos comparado con el abono líquido de bovino $(0.17 \%)$ y abono líquido de cerdo $(0.05$ $\%)$ [42]. Con respecto al contenido de potasio, el mucilago de cacao tiene valores altos $(0.2 \%)$ respecto de biol fresco $(0.06 \%)$ [40], valores bajos comparado con biol supermagro (112 ppm) [41] y valores altos comparado con biol de bovino $(0.06 \%)$ y biol de cerdo $(0.04 \%)$ [42].

Tabla 4. Caracterización de los mucilagos de cacao y copoazú.

\begin{tabular}{lcc}
\hline Parámetros & Cacao & Copoazú \\
\hline Humedad (\%) & $83.38 \pm 0.41 \mathrm{a}$ & $94.4 \pm 0.27 \mathrm{~b}$ \\
Materia seca (\%) & $16.62 \pm 0.35 \mathrm{~b}$ & $5.60 \pm 0.40 \mathrm{a}$ \\
Acidez titulable (ácido cítrico, \%) & $0.66 \pm 0.01 \mathrm{a}$ & $2.77 \pm 0.02 \mathrm{~b}$ \\
Peso específico & 1.066 & 1.026 \\
pH & $3.55 \pm 0.01 \mathrm{a}$ & $4.85 \pm 0.01 \mathrm{~b}$ \\
${ }^{\circ}$ Brix $\left(20^{\circ} \mathrm{C}\right)$ & $15.97 \pm 0.03 \mathrm{~b}$ & $9.37 \pm 1.23 \mathrm{a}$ \\
Conductividad eléctrica $\left(\mathrm{mS} \mathrm{cm}^{-1}\right)$ & $2.79 \pm 0.02 \mathrm{a}$ & $7.82 \pm 0.73 \mathrm{~b}$
\end{tabular}




$\begin{array}{lcc}\text { Cenizas }(\%) & 15.73 \pm 0.998 \mathrm{~b} & 1.87 \pm 0.001 \mathrm{a} \\ \text { Fosforo }\left(\mathrm{mg} \mathrm{L}^{-1}\right) & 11.18 \pm 0.49 \mathrm{~b} & 3.59 \pm 0.82 \mathrm{a} \\ \text { Potasio }\left(\% \mathrm{p} \mathrm{v}^{-1}\right)^{\mathrm{a}} & 0.2 & 0.4 \\ \text { Nitrógeno }(\%) & 0.08 \pm 0.0003 \mathrm{a} & 0.12 \pm 0.0082 \mathrm{~b} \\ \text { Carbono }(\%) & 1.98 \pm 0.03 \mathrm{~b} & 1.51 \pm 0.03 \mathrm{a} \\ \mathrm{n}=3 .{ }^{\mathrm{a}} \mathrm{n}=2 . \text { Letra minúscula diferente en la misma fila indica diferencia significativa. Nivel de significancia } 0.05 .\end{array}$

\subsection{Respuesta agronómica}

Para el ensayo de respuesta agronómica se usó el mucilago de cacao dado su mayor rendimiento (Tabla 3).

\section{Tasa Absoluta de Crecimiento}

Las hojas de maíz utilizan el $\mathrm{CO}_{2}$ para elaborar fotoasimilados usando un mecanismo fotosintético $\mathrm{C}_{4}$ en su estado vegetativo (V) mientras que en su estado reproductivo (R) se puede adicionalmente, llevar a cabo una fijación parcial de $\mathrm{CO}_{2}$ en la cáscara mediante un mecanismo fotosintético $\mathrm{C}_{3}$ [43]. Al evaluar la tasa absoluta de crecimiento en la variedad ICA V-305 se observó, similar efecto entre los 21 a 28 dds en el control y los tratamientos, con una tendencia creciente hasta los $36 \mathrm{dds}$, donde los tratamientos con $10 \%$ y 15 $\%$ de mucilago obtuvieron los valores máximos de TAC con 2.72 y $1.54 \mathrm{~g} \mathrm{día}^{-1}$, respectivamente. Es de notar, que, en los 36 días de siembra, la planta de maíz se encontraba en etapa vegetativa V10-V12, en donde requería altas cantidades de nutrientes siendo insuficientes los nutrientes que proporcionaban estos abonos foliares evaluados ya que se observó una disminución en la TAC siendo una etapa (V10-V12) donde la planta inicia un incremento acelerado en la acumulación de foto asimilados [44]. Un comportamiento similar se observó en estudios de maíz solo y asociado con pastos (Brachiaria cv. mulato, Brachiaria brizantha (Hochst. ex A. Rich.) Stapf cv. toledo y Brachiaria decumbens Stapf) donde el mayor aumento de masa seca fue a los 30 días para disminuir la TAC a los 42 dds [45], en estudios de respuesta agronómica de Cucurbita pepo L. con valor máximo de TAC (6.5 $\left.\mathrm{g} \mathrm{dia}^{-1}\right)$ a los 62 dds [46] y en estudios de desempeño agronómico en maíz usando bioestimulantes con un valor máximo de TAC de $0.9 \mathrm{~g}_{\text {dia }}{ }^{-1}$ entre 27 a 36 días [47]. Otro estudio de respuesta agronómica de maíz bajo diferentes regímenes hídricos y en un período de estudio de 107 días mostró un comportamiento de la TAC de tipo campana de Gauss con un valor máximo de TAC de $75 \mathrm{~g} \mathrm{~m}^{-2}$ $\mathrm{dia}^{-1}$ a los 67 días de estudio [48]. Es de notar que el tratamiento con $5 \%$ mucilago mostró su valor máximo (1.91 $\left.\mathrm{g} \mathrm{dia}^{-1}\right)$ a los 42 dds mostrando un efecto más lento en la producción de biomasa, similar al control y fertisun (Figura 3). En los valores de TAC de variedad Calilla todos los tratamientos mostraron una tendencia creciente similar registrando sus valores máximos a los 42 dds, donde el $10 \%$ de mucilago presentó el valor más alto (Figura 4). Es de notar que los valores de TAC de maíz variedad Calilla (Figura 4) fueron ligeramente mayores respecto de los valores TAC de maíz de la variedad ICA V-305 (Figura 3). La tendencia siempre creciente de TAC ha sido observada en papaya en un periodo de estudio de 95 días [49]. En cultivo de café se observó que al aumentar el nivel de irrigación con agua, hubo un aumento en forma polinomial de la TAC y TRC [50]. Al evaluar la respuesta agronómica de cultivares de maíz (P-3051 y Br-5011) sometidos a estrés salino $\left(\mathrm{NaCl}, 0-100 \mathrm{~mol} \mathrm{~m}^{-2}\right)$ se observó que la TAC y la TRC disminuyeron durante todo el tiempo de estudio al aumentar la concentración salina [51]. 


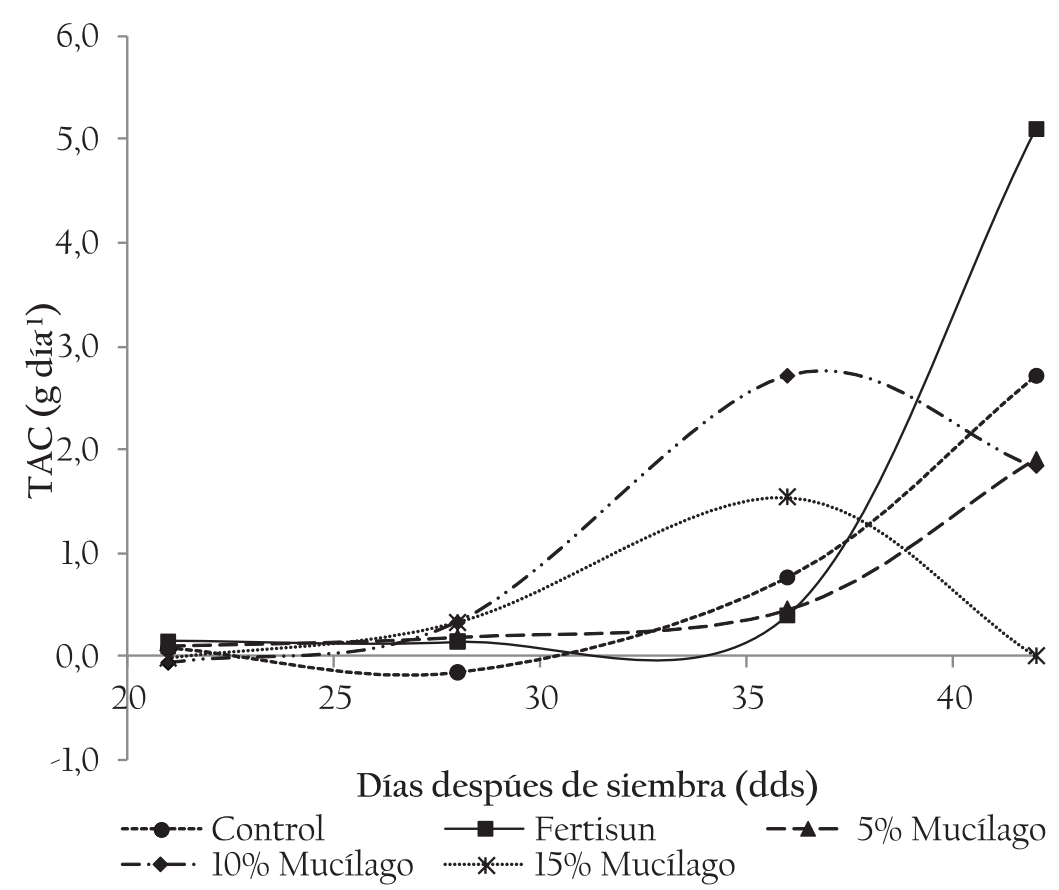

Figura 3. Comportamiento de la Tasa Absoluta de Crecimiento (TAC) en plantas de maíz de acuerdo al efecto de abonos foliares en variedad ICA V-305.

Al evaluar el efecto de Rottboellia cochinchinensis (Lour.) Clayton, como maleza sobre el valor de TAC en cultivo de maíz ICA V-109 se observó que en presencia entre 2-8 plantas de maleza $\mathrm{m}^{-2}$ el valor de TAC de cultivo de maíz presento una tendencia similar a la registrada para maíz ICA V-305 del presente estudio (Figura 3) indicando que la TAC es poco sensible a efectos de maleza, sin embargo se observó además que transcurrido el $50 \%$ del periodo de estudio (99 días) la maleza presentó su mayor crecimiento simultáneo con el inicio de la floración y llenado del grano de maíz, lo que implicaría disminución en foto asimilados para el maíz [52].

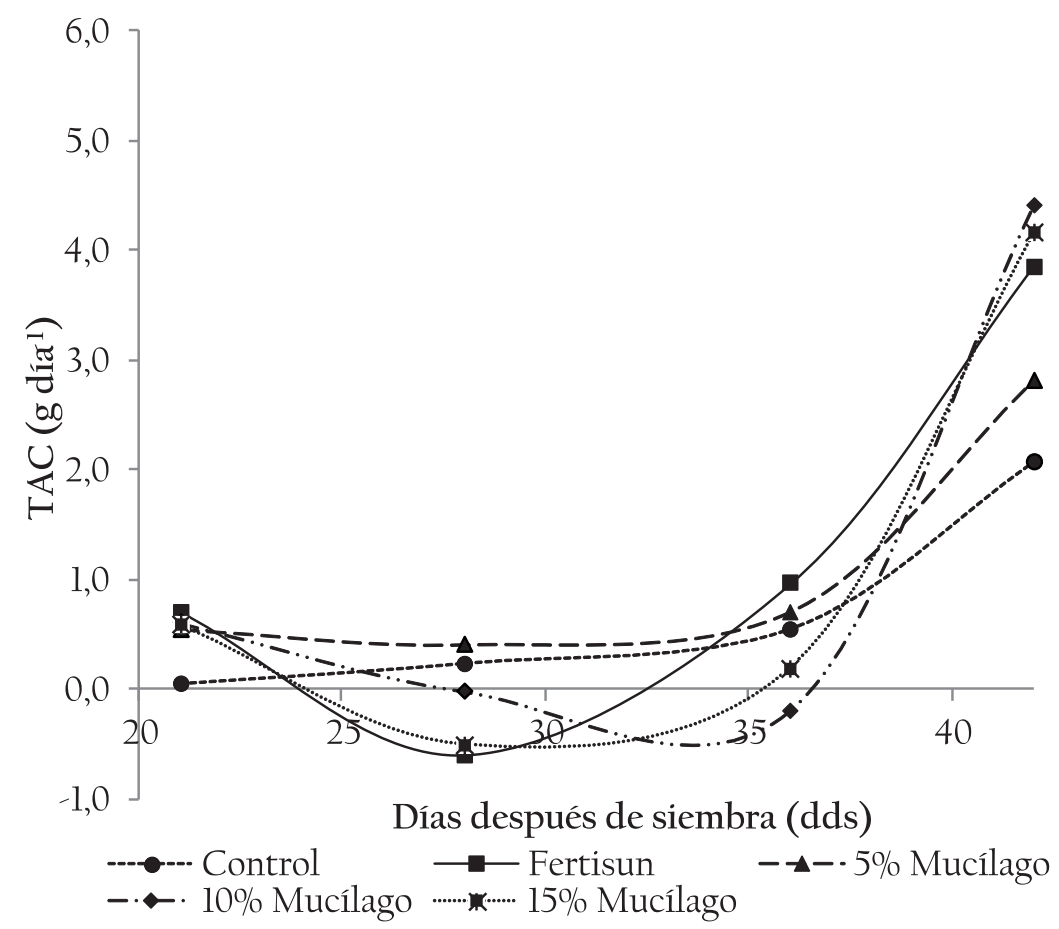

Figura 4. Comportamiento de la Tasa Absoluta de Crecimiento (TAC) en plantas de maíz de acuerdo al efecto de abonos foliares en variedad Calilla. 
Aunque la tasa de crecimiento absoluto (TAC) indica la velocidad de crecimiento de la planta, es mejor expresar la tasa de crecimiento, según el propio peso de la planta. En este caso, se trata de la tasa de crecimiento relativa, ya que conceptualmente el análisis de crecimiento establece que la tasa de crecimiento de una planta o de cualquier órgano de ella es una función del tamaño inicial. Esto indica que esta medida puede ser más precisa, ya que considera el material asignado sobre el material ya existente, de forma que sea proporcional al tamaño de la planta y su capacidad fotosintética [49].

La breve duración de los máximos valores de la TAC para la variedad ICA V-305, concuerda con Sedano, et al. [46] quienes señalan que la TAC puede alcanzar un valor máximo en un tiempo corto o mantenerse por un periodo mayor, según la especie. Sin embargo, se observó que en la variedad Calilla, los resultados obtenidos en la TAC aumentan con relación del peso seco manteniendo su incremento en periodos mayores [53], de igual forma, Hernández y Soto [54] encontraron que los valores máximos de la TAC en maíz se alcanzaron en períodos tempranos de la fase reproductiva.

\section{Tasa Relativa de Crecimiento}

En el caso de maíz variedad ICA V-305, los valores de TRC de los tratamientos $10 \%$ y 15 $\%$ de mucilago presentaron forma de campana de Gauss con valores máximos a los 35 dds, mientras que el tratamiento de $5 \%$ mucilago presento un ligero aumento hasta los $42 \mathrm{dds}$ (Figura 5). En el caso de los valores de TRC para maíz variedad Calilla se observó una disminución en los tratamientos 5, 10 y $15 \%$ de mucilago, sin embargo entre los 28 a 36 dds, el tratamiento de $15 \%$ mucilago presento un fuerte incremento del valor TRC hasta los 42 dds en forma similar al tratamiento de $10 \%$ mucilago, los cuales presentaron valores máximos mayores a fertisun (Figura 6). Esta respuesta en los valores TRC de las variedades de maíz evaluadas en el presente estudio puede estar relacionada con lo planteado por Criollo y García [55], quienes exponen que el crecimiento suele tener una dinámica exponencial y suele reflejar diferencias significativas entre especies o manejo agronómico diferente, en este caso, las variedades de maíz implementadas. Por otra parte, Gil y Miranda [56] plantean que para la TRC existe correlación entre el proceso de crecimiento y desarrollo de diversas partes de la planta, que puede explicarse en términos del suministro y la demanda.

La disminución inicial de TCR en maíz de variedad ICA y Calilla hasta 28 dds es similar a lo registrado para papaya, lo cual está de acuerdo con lo esperado, ya que cualquier incremento en peso, altura o área foliar a lo largo de un determinado período está directamente relacionado con el tamaño alcanzado en el período anterior [49]. Al observar el efecto de maleza sobre el valor de TRC en cultivo de maíz ICA-V-109 (Rottboellia cochinchinensis) se estableció que el cultivo de maíz ICA-V-109 sin maleza presentó una disminución del valor TRC a lo largo del tiempo de estudio (99 días), tendencia que varió hacia una forma de campana de Gauss al aumentar la densidad de malezas ( 2 a 8 plantas maleza $\mathrm{m}^{-2}$ ) en el cultivo de maíz ICA-V-109 [52], lo cual videncia que la presencia de maleza o arvenses afecta el comportamiento gráfico de los datos de TRC, como sucedió en el presente estudio para maiz-V-305 (Figura 5). Es de notar, que el comportamiento decreciente de la TRC en estudios de respuesta agronómica de cultivos, como en el caso de Cucurbita pepo L. [46], se asocia a la no presencia de malezas (arvenses) que generen competencia con el cultivo como indicaron Rivera, et al. [52]. Una situación similar se observó en el estudio de Santos, et al. [47] al evaluar el desempeño agronómico de maíz usando bioestimulantes donde se observó una disminución permanente de los valores de TRC durante 45 días después de germinación. Otro estudio de respuesta agronómica de maíz bajo diferentes regímenes hídricos y en un período de estudio de 107 días mostró un comportamiento decreciente de la TRC con un valor máximo inicial de 0.16 g día $^{-1}$ [48]. Los resultados obtenidos para TRC en el presente estudio son similares a los obtenidos para respuesta agronómica de maíz solo $\mathrm{y}$ asociado a pastos (B. brizantha, $B$. cv. Mulato y $B$. decumbens) donde el valor TRC fue máximo a los 30 dds y disminuyó posteriormente. El anterior estudio evidencio que el lento crecimiento inicial de los pastos hizo que el maíz se desarrollara sin competencia [45]. 


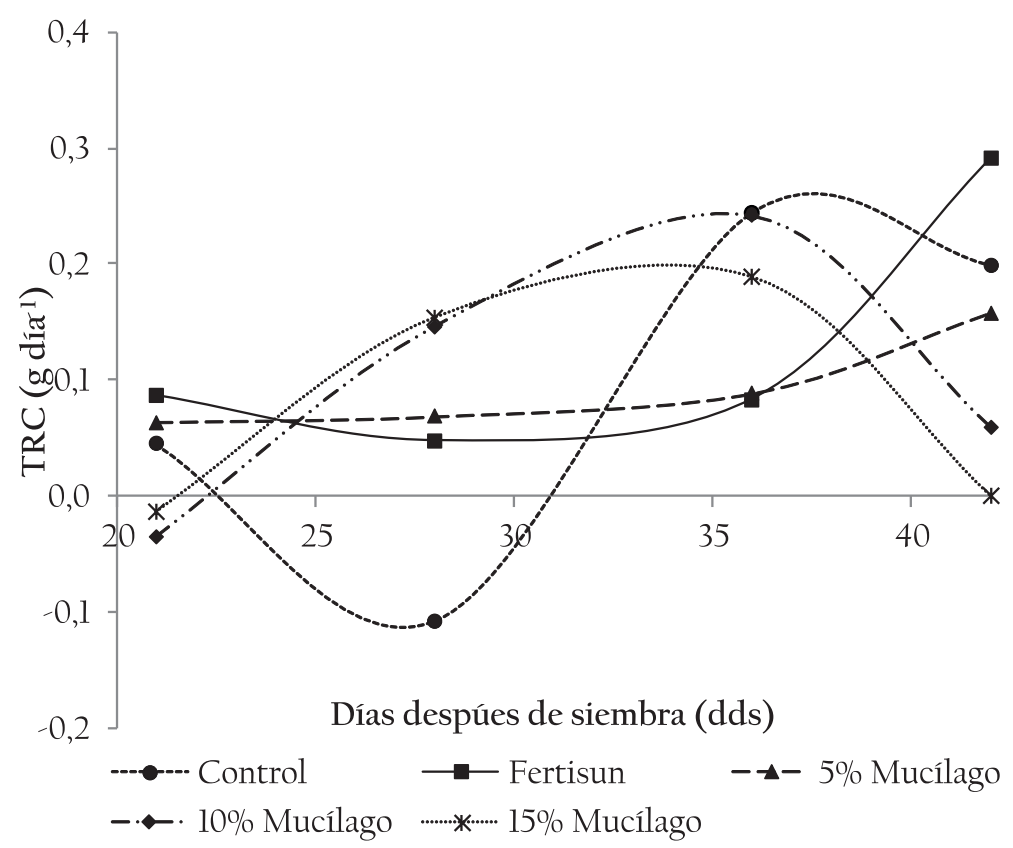

Figura 5. Comportamiento de la Tasa Relativa de Crecimiento (TRC) en plantas de maíz de acuerdo al efecto de abonos foliares. Variedad ICA V-305.

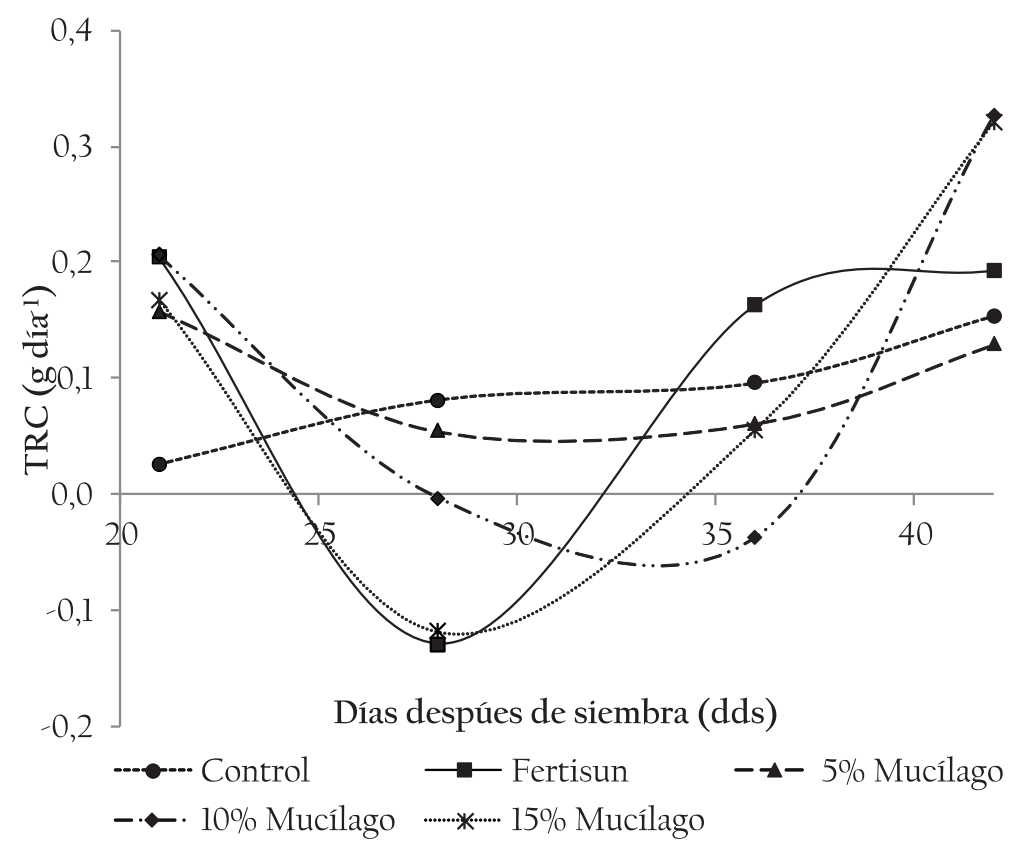

Figura 6. Comportamiento de la Tasa Relativa de Crecimiento (TRC) en plantas de maíz de acuerdo al efecto de abonos foliares Variedad Calilla.

\subsection{Aspectos relevantes}

Al comparar los parámetros de madurez de las pulpas de los frutos evaluados, se estableció diferencia significativa $(\alpha=0.05)$ en $\mathrm{pH}$, acidez titulable y ${ }^{\circ}$ Brix. El maraco presentó el mayor valor de índice de madurez (Tabla 3). El fruto con mayor potencial comercial de producción de mucilago fue el cacao (mezcla de variedades rojo y amarillo) ya que presentó el mayor rendimiento correspondiente a $4.5 \mathrm{ml}$ mucilago $\mathrm{kg}^{-1}$ grano fresco hora ${ }^{-1}$, cuyo valor es el doble del mucilago producido por el copoazú y 22.5 veces más del mucilago que produjo el maraco (Tabla 4). El mucilago de cacao obtenido luego de escurrimiento del grano mostró contenidos de $11.18 \mathrm{mg} \mathrm{P} \mathrm{L}^{-1}$, potasio de $0.2 \% \mathrm{~K}$ y $0.08 \% \mathrm{~N}$. En la evaluación de TAC de maíz en dos variedades se observó que la variedad Calilla presentó mayor crecimiento en menor tiempo cuando se 
aplicó mucilago al $5 \%$ respecto de la variedad ICA V- 305 (Figura 3 y 4). Es de notar, que la aplicación de $5 \%$ de mucilago al maíz mostro siempre valores positivos de TAC, indicando poca variabilidad entre especímenes evaluados, contrario a lo observado para 10 y $15 \%$ de mucilago aplicado. Lo anterior indicaría que el tratamiento al $5 \%$ de mucilago favoreció en forma más adecuada (menos variabilidad en valores TAC) el crecimiento de maíz variedad Calilla, el cual es la variedad criolla que se ha tratado de implementar en la región de piedemonte de la Amazonia colombiana y además fue evidente una mayor ganancia de materia seca en Calilla (2.82 g día ${ }^{-1}$ ) (Figura 3) respecto de ICA V305 (1.91 $\mathrm{g} \mathrm{día}^{-1}$ ) (Figura 4). Considerando la existencia de malezas en el sitio de estudio, las cuales fueron retiradas solo en los dos primeros muestreos (13 y $21 \mathrm{dds}$ ) no se observó una tendencia clara en los valores TRC para las dos variedades de maíz evaluadas.

\section{CONCLUSIONES}

El maraco presentó el mayor valor de índice de madurez de las pulpas de los frutos evaluados. El fruto con mayor potencial comercial de producción de mucilago fue el cacao ya que presentó el mayor rendimiento $(4.5 \mathrm{ml}$ mucilago $\mathrm{kg}^{-1}$ grano fresco hora-1), El mucilago de cacao obtenido luego de escurrimiento del grano mostró contenidos de $11.18 \mathrm{mg} \mathrm{P} \mathrm{L}^{-1}$, potasio de $0.2 \% \mathrm{~K}$ y $0.08 \%$ N. En la evaluación de TAC de maíz en dos variedades se observó que la variedad Calilla presentó mayor crecimiento en menor tiempo cuando se aplicó mucilago al $5 \%$ respecto de la variedad ICA V- 305, por lo cual se consideró el mejor tratamiento para usar como abono líquido. Considerando la existencia de malezas en el sitio de estudio, las cuales fueron retiradas solo en los dos primeros muestreos ( 13 y $21 \mathrm{dds}$ ) no se observó una tendencia clara en los valores TRC para las dos variedades de maíz evaluadas.

\section{AGRADECIMIENTOS}

A la Vicerrectoría de Investigaciones de la Universidad de la Amazonia. A la Universidad Surcolombiana por los análisis de absorción atómica de potasio. Al ingeniero agroecólogo David Castillo por su apoyo en el trabajo en campo.

\section{REFERENCIAS}

[1] R. O. O. Ortiz, V. R. A. Gallardo, and J. M, Rangel. Applying life cycle management of Colombian cocoa production. Food Science and Technology, vol. 34, no 1, pp. 62-68, http://dx.doi.org/10.1590/S010120612014005000006 , Mar. 2014.

[2] C. R. G. García, A. Perdomo, O. Ortiz, P. Beltrán, and K. López. Characterization of the supply and value chains of Colombian cocoa. DYNA, vol. 81, no. 187, pp. 3040, http://dx.doi.org/10.15446/dyna. v81n187.39555, Oct. 2014.

[3] R. Martínez, P. Torres, M. A. Meneses, J. G. Figueroa, J. A. Pérez-Álvarez, M. Viuda-Martos. Chemical, technological and in vitro antioxidant properties of cocoa (Theobroma cacao L.) co-products. Food Research International, vol. 49, no. 1, pp. 39-45, https://doi.org/10.1016/j. foodres.2012.08.005, Nov. 2012.

[4] H. Barazarte, E. Sangronis, y E. Unai. La cáscara de cacao (Theobroma cacao L.): Una posible fuente comercial de pectinas. $A L A N$ [online], vol.58, no. 1, pp. 64-70, Mar. 2008.

[5] F. M. Castillo, H. M. Ramírez, G. R. García, G. M. Bernal, A. B. Espinosa, F. J. A. Solís, y B. C. Durán. Reaprovechamiento integral de residuos agroindustriales: cáscara y pulpa de cacao para la producción de pectinas. Revista Latinoamericana el Ambiente y las Ciencias, vol. 1, no. 2, pp. 45-66, Ago. 2010.

[6] Z. Kalvatchev, D. Garzaro, y C. F. Guerra. Theobroma cacao L.: un nuevo enfoque para nutrición y salud. Agroalimentaria, vol. 4, no. 6, pp. 23-25, Jun. 1998.

[7] CdSO Nunes, GBM de Carvalho, MLC da Silva, GP da Silva, BAS Machado and APT Uetanabaro. Cocoa pulp in beer production: Applicability and fermentative process performance. PLoS ONE, vol. 12, no. 4, e0175677, https://doi.org/10.1371/journal. pone.0175677, Abr. 2017. 
[8] T. C. A. Vallejo, D. R. Ocampo, R. W. Morales, V. R. Soria, C. J. F. Vera, C. C. Baren. Utilización del mucílago de cacao, tipo nacional y trinitario, en la obtención de jalea. ESPAMCIENCIA, vol. 7, no. 1, pp. 51-58, May. 2016.

[9] E. Y. Arteaga. Estudio del desperdicio del mucilago de cacao en el canton naranjal (provincia del guayas). Revista ECA Sinergia. Facultad de Ciencias Administrativas y Económicas. U.T.M. vol. 4, no. 1, pp. 49-59, Dic. 2013.

[10] Reglamento Técnico de Fertilizantes y Acondicionadores de Suelos para Colombia, Resolución 00150 del ICA, 2003.

[11] Productos para la industria agrícola. Productos orgánicos usados como abonos o fertilizantes y enmiendas o acondicionadores del suelo, Norma Técnica Colombiana 5167, 2011.

[12] Reglamento para la producción primaria, procesamiento, empacado, etiquetado, almacenamiento, certificación, importación, comercialización y se establece el Sistema de Control de Productos Agropecuarios Ecológicos, Resolución 000187 del Ministerio de Agricultura y Desarrollo Rural, 2006.

[13] Requisitos para el registro de departamentos técnicos de ensayos de eficacia, productores e importadores de bioinsumos de uso agrícola y se dictan otras disposiciones. Resolución 000698 del ICA, 2011.

[14] V. K. L. Ortiz, L. R. Álvarez. Efecto del vertimiento de subproductos del beneficio del cacao (Theobroma cacao L.) sobre algunas propiedades químicas y biológicas en los suelos de una finca cacaotera, municipio de yaguará (Huila, Colombia). Boletín Científico. Museo de Historia Natural, vol. 19, no. 1, pp 65-84, http:// dx.doi.org/10.17151/bccm.2015.19.1.5, Ene. 2015.

[15] H. M. R. Hipo. Aplicación de mucilago de semillas de cacao (Theobroma cacao
L.) en el control de malezas. Trabajo de grado. Facultad de ciencias agropecuarias. Universidad Técnica de Ambato. Ambato. 2017.

[16] P. Mamani, E. Chávez y N. Ortuño. El Biol: Biofertilizante casero para la producción ecológica de cultivos [online]. Biblioteca Virtual Agrícola. 2015. Disponible en: http:// www.proinpa.org/tic/pdf/Bioinsumos/Biol/ pdf59.pdf.

[17] N. L. Betancourt, D. A. Castillo, J. E. Velásquez y J. Muñoz. Efecto del carbón vegetal y del abono orgánico en la producción de maíz (Zea mays L.) como aproximación a la formación de terras pretas en el piedemonte amazónico colombiano. Momentos de Ciencia, vol. 6 no. 2, pp 85-90, May. 2015.

[18] M. Bhanti and A. Taneja. Contamination of vegetables of different seasons with organophosphorous pesticides and related health risk assessment in northern India. Chemosphere, vol. 69, no. 1, pp 63-68. https://doi.org/10.1016/j. chemosphere.2007.04.071, Ago. 2007.

[19] J. Braudeau. El cacao. Técnicas agrícolas y producciones tropicales. Barcelona, España. Editorial Blumé. 1970. 297 pp.

[20] J. Vera, C. Vallejo, D. Parraga, J. Macías, R. Ramos y W. Morales. Propiedades físicoquímicas y sensoriales de las almendras de quince clones de cacao Nacional (L.) en el Ecuador. Revista Ciencia y tecnología vol. 7, no. 2, pp. 21-34, Jul. 2014.

[21] J.A. Oladejo and M. O. Adetunji. Economic analysis of maize (Zea mays L.) production in Oyo state of Nigeria. Agricultural Science Research Journals, vol. 2, no. 2, pp. 77-83, Feb. 2012.

[22] E. Trujillo, D. F. Castro y J. A. Morales. Inventario de la familia Araceae en la granja agroecológica Balcanes de la Universidad de la Amazonia. Momentos de Ciencia, vol. 11, no. 1, pp. 17-22, Sep. 2016. 
[23] D. H. Ruiz, N. A. Obregón y N. C. Arriaga. Diversidad de anuros en humedales del Centro de Investigación Amazónica Macagual, Florencia, Caquetá, Colombia. Biodiversidad Neotropical, vol. 4, no. 1, pp. 42-48. http://dx.doi.org/10.18636/ bioneotropical.v4i1.159.g110. Ene. 2014.

[24] R. P. A. García, P. W. Rodríguez y E. K. Chalarca, Z. A. Andrade, Estudio microbiológico y fisicoquímico de hongos comestibles (Pleurotus ostreatus y Pleurotus pulmonarius) frescos y deshidratados. Ingenierias \& Amazonia, vol. 7, no. 1, pp. 41-47. Ago. 2014.

[25] A. A. Rodríguez. Estudio de tres métodos para la obtención de pulpa de cacao (Theobroma cacao variedad CCN-51). Trabajo de grado. Facultad de ingeniería y ciencias agropecuarias. Universidad de la Américas. Quito. 2013.

[26] O. R. E. Verbel, M. I. D. Mercado y G. L. E. Montes. Remoción de la turbidez del agua del rio Magdalena usando el mucilago del nopal Opuntia ficus-indica. Producción + limpia, vol. 18. no. 1, pp. 19-27, Ene. 2013.

[27] E. O. Afoakwa, J. Quao, J. Takrama, A. S. Budu and F. K. Saalia. Chemical composition and physical quality characteristics of Ghanaian cocoa beans as affected by pulppre-conditioning and fermentation. Journal of food science and technology, vol. 50, no. 6, pp. 1097-1105. https://doi.org/10.1007/s13197-011-04465, Dic. 2013.

[28] S. S. Nielsen. Food analysis. Purdue University. Fourth edition. Springer. 2010.

[29] M. Bahadori and H. Tofighi. A Modified Walkley-Black Method Based on spectrophotometric Procedure, Communications in Soil Science and Plant Analysis, vol. 47, no. 2, pp. 213-220, http:// dx.doi.org/10.1080/00103624.2015.111811 8, Ene. 2016.
[30] A.M Zamudio, M. L. Carrascal, C. E., Pulido, J. F. Gallardo, E. A. Ávila, M. A. Vargas y D. F. Vera. Métodos analíticos del laboratorio de suelos. Sexta edición. Instituto Geográfico Agustín Codazzi IGAC. 2006.

[31] J. Di Rienzo, F. González, L. Tablada, P. Díaz, C. Robledo y M, Balzirini. Estadística para las ciencias agropecuarias. Séptima edición. Editorial Brujas. 2009.

[32] P. Almanza, P. Serrano y O. Castro. Respuesta Fisiológica del lulo (Solanun quitoense Lam.) a la fertilización orgánica en Tinjacá, Boyacá. Cultura Científica, vol. 6, no. 6, pp. 82-86, Oct. 2008.

[33] E, N. Escobar, D. J. Mora y J. N. Romero. Respuesta agronómica de Zea mays L. y Phaseolus vulgaris L. a la fertilización con compost. Revista Luna Azul, no. 37, pp. 1829, Jul. 2013.

[34] M. Aristizábal. Evaluación del crecimiento y desarrollo foliar del plátano hondureño enano (Musa $\mathrm{AAB}$ ) en una región cafetera colombiana. Agronomía, vol. 16, no. 2, pp. 23-30, Sep. 2008.

[35] Instituto de Hidrología, Meteorología y Estudios Ambientales (IDEAM). Reporte climático [on line]. 2015. Disponible en: http://www.ideam.gov.co/

[36] B. M. P. Carrillo, J. J. E Cardona, S. R. O. Díaz, D. L. L. Orduz, R. L. F. Peña, G. M. S. Hernández y L. E. Mosquera. Los ingredientes naturales de la Amazonia colombiana: sus aplicaciones y especificaciones técnicas. Instituto Amazónico de Investigaciones CientíficasSinchi, Universidad Nacional de Colombia, 112 pp. 2017.

[37] L. M. Melgarejo, M. S. Hernández, J. A. Barrera,M.Carrillo.Ofertay potencialidades de un banco de germoplasma del género Theobroma en el enriquecimiento de los sistemas productivos de la región amazónica. Instituto Amazónico de Investigaciones Científicas- Sinchi, Universidad Nacional de Colombia, 225 pp. 2006. 
[38] C. Romero y A. Zambrano. Análisis de azúcares en pulpa de cacao por colorimetría y electroforesis. Revista Científica UDO Agrícola, vol. 12, no. 4, pp. 906-913, Dic. 2012.

[39] H. Dziadowiec. The decomposition of plant litter fall in an oak-linden-hornbeam forest and an oak-pine mixed forest of the Bialwieża national park. Acta societatis botanicorum poloniae, vol. 56, no. 1 , pp. 169-185, https://doi.org/10.5586/ asbp.1987.019, Ene. 1987.

[40] L. Warnar y H. Oppenoorth. El Biol: el fertilizante supremo: estudio sobre el biol, sus usos y resultados. Hivos, 47 pp. 2014.

[41] N. Aliaga. Producción de biol supermagro. Cedepas norte. Centro ecuménico de promoción y acción social, 9 pp. s.f.,

[42] Sistema biobolsa. Manual del biol [online]. Disponible en: http://sistemabiobolsa.com/ pdf/manualDeBiol.pdf. s.f.

[43] J. J. L. Pengelly, S. Kwasny, S. Bala, J. R. Evans, E. V. Voznesenskaya, N. K. Koteyeva, G. E. Edwards, R. T. Furbank and S. Von Caemmerer. Functional Analysis of Corn Husk Photosynthesis. Plant Physiology, vol. 156, pp. 503-513, https:// doi.org/10.1104/pp.111.176495, Jun. 2011.

[44] A. Fassio, A. I. Carriquiry, C. Tojo y R. Romero. "Maíz: Aspectos sobre fenología". Unidad de difusión e información tecnológica del INI, Uruguay, Serie técnica no. 101, 1998.

[45] A. Rincón, G. A. Ligarreto y D. Sanjuaneto. Crecimiento del maíz y los pastos (Brachiaria sp.) establecidos en monocultivo y asociados en suelos ácidos del piedemonte llanero colombiano. Agronomía Colombiana, vol. 25, no. 2, pp. 264-272, Jul. 2007.
[46] C. G. Sedano, H. V. A. González, E. M. Engleman y V. C. Villanueva. Dinámica del crecimiento y eficiencia fisiológica de la planta de calabacita. Revista Chapingo Serie Horticultura, vol. 11, no. 2, pp. 291297, Ene. 2005.

[47] V. M. dos Santos, A. V. Melo, S. C. de, Siebeneichler, D. P. Cardoso, L. P. F. Benício and M. A. F. Varanda. Physiological indices of seedlings of maize (Zea mays L.) under the action of biostimulants. Journal of Biotechnology and Biodiversity, vol. 4, no. 3, pp. 232-239, Ago. 2013.

[48] . Garcia, R. G. B. André, J. A. Galbiatti e S. Tannous. Análise de crescimento de uma cultura de milho submetida a diferentes regimes hídricos. Nucleus, vol. 5, no. 1, pp. 239-251, Mar. 2008.

[49] J. F. de Lima, C. P. Peixoto e C. A. da Silva. Índices fisiológicos e crescimento inicial de mamoeiro (Carica papaya L.) em casa de vegetação. Ciência e Agrotecnologia, vol. 31, no. 5, pp. 1358-1363, https://doi. org/10.1590/S1413-70542007000500013, Mar. 2007.

[50] A. J. Souza, R. J. Guimarães, A. Colombo, J. A. Sant'Ana and D. T. Castanheira. Quantitative analysis of growth in coffee plants cultivated with a water-retaining polymer in an irrigated system. Revista Ciência Agronômica, vol. 47, no. 1, pp. 162-171 http://dx.doi.org/10.5935/18066690.20160019, Mar. 2016.

[51] A. D. Neto e J. N. Tabosa. Estresse salino em plântulas de milho: parte I análise do crescimento. Revista Brasileira de Engenharia Agrícola e Ambiental, vol. 4, no. 2, pp. 159-164, Ene. 2000.

[52] J. A. Rivera, R. E. Cabrales, R. A. Montoya. Efecto de la densidad poblacional de caminadora (Rottboellia cochinchinensis (L.) Sw. Clayton) sobre el cultivo de maíz ICA V- 109. Temas agrarios, vol. 12, no. 2, pp. 51 - 61, https://doi.org/10.21897/rta. v12i2.657, Jul 2007. 
[53] M. C. Arenas, J. E. Vélez y J. H. Camacho. Crecimiento del fruto de peral bajo el sistema de riego por goteo con una y dos líneas. Revista Colombiana de Ciencias Hortícolas, vol. 6, no. 2, pp. 140-151, https:// doi.org/10.17584/rcch.2012v6i2.1972, Jul. 2012.

[54] N. Hernández y F. Soto. Influencia de tres fechas de siembra sobre el crecimiento y la relación fuente-demanda del cultivo del maíz (Zea mays L.). Cultivos Tropicales, vol. 33, no. 1, pp. 28-34, Ene. 2012.
[55] H. Criollo y J. García. Efecto de la densidad de siembra sobre el crecimiento de plantas de rábano (Raphanus sativus L.) bajo invernadero. Revista Colombiana de Ciencias Hortícolas, vol. 3, no. 2, pp. 210-222 https://doi.org/10.17584/ rcch.2009v3i2.1214, Jul. 2009.

[56] A. I. Gil y D. Miranda. Efecto de cinco sustratos sobre índices de crecimiento de plantas de papaya (Carica papaya L.) bajo invernadero. Revista Colombiana de Ciencias Hortícolas, vol. 1, no. 2, pp. 142-153, https://doi.org/10.17584/ rcch.2007v1i2.1156, Jul. 2007. 